\title{
Mechanism of the inhibition of the STAT3 signaling pathway by EGCG
}

\author{
YANG WANG $^{1}$, XUEQUN REN $^{1}$, CHAOYANG DENG $^{2}$, LU YANG $^{2}$, ERFU YAN $^{3}$, \\ TAO GUO ${ }^{4}$, YANMIN $\mathrm{LI}^{5}$ and MARVIN XUEJUN XU ${ }^{6}$
}

\author{
${ }^{1}$ Huaihe Hospital, Henan University, Kaifeng 475000; ${ }^{2}$ Shanghai McAry Biomedical Technology Co. Ltd., \\ Zhangjiang Hi-Tech Park, Pudong New Area, Shanghai 201203; ${ }^{3}$ Department of Pharmacy, \\ Tianjin University, Tianjin; ${ }^{4}$ Center for Drug Delivery System, Shanghai Institute of Materia Medica, \\ State Key Laboratory of Drug Research, Chinese Academy of Sciences, Shanghai 201203; \\ ${ }^{5}$ Department of Physics, Shangqiu Normal College, Shangqiu 476000; ${ }^{6}$ Department of Pharmacology, \\ Basic Medical College, Zhengzhou University, Zhengzhou, Henan 450001, P.R. China
}

Received May 1, 2013; Accepted June 25, 2013

DOI: $10.3892 /$ or.2013.2743

\begin{abstract}
Signal transducer and activator of transcription 3 (STAT3) is an oncogene that promotes cell survival, proliferation, and motility. In the present study, we explored the mechanism involved in the inhibition by epigallocatechin3-gallate (EGCG) of STAT3 signaling as detected by surface plasmon resonance (SPR)-binding assays and in silico docking. Stat3-binding assay indicated that EGCG significantly interrupted Stat 3 peptide binding at micromolar concentrations, and the docking experiments indicated that EGCG had a strong interaction with Arg-609, one of the key residues in the STAT3 $\mathrm{SH} 2$ domain that contributes greatly to Stat 3 and phosphorylated peptide binding. Following treatment of the hepatocellular carcinoma cell lines BEL-7402 and QGY-7703 with EGCG, in vitro, EGCG significantly suppressed cell proliferation as detected by MTT assay, induced apoptosis as detected by flow cytometry, dramatically lowered the expression levels of phosphorylated Stat 3 proteins (p-Stat3) as determined by immunoblot detection, and inhibited the expression of multiple genes including Bcl-xL, c-Myc, VEGF and cyclin D1 as demonstrated by RT-PCR analysis. In conclusion, our research data indicate that the anticancer function of green tea results from the inhibition of the STAT3 signaling pathway by EGCG.
\end{abstract}

Correspondence to: Dr Marvin Xuejun Xu, Department of Pharmacology, Basic Medical College, Zhengzhou University, 100 Kexue Road, Zhengzhou, Henan 450001, P.R. China

E-mail: xxjun@zzu.edu.cn

Key words: epigallocatechin-3-gallate, signal transducer and activator of transcription 3, hepatocellular carcinoma, molecular docking

\section{Introduction}

Signal transducer and activator of transcription 3 (STAT3) is one of the seven members of the Stat protein family that mediates the actions of many cytokines and growth factors. STAT3 shows constitutive activity in many different types of cancers, including breast, prostate, head and neck, lung, colon, liver and pancreatic cancers, and large granular lymphocytic leukemia and multiple myeloma (1-3). In addition, human tumor xenograft studies in mice have repeatedly demonstrated that inhibition of STAT3 signaling results in decreased tumor growth and improved animal survival by inducing apoptosis in tumor cells, inhibiting angiogenesis (4), and enhancing antitumor immune-mediated cytotoxicity $(1,5)$. Thus, STAT3 has been identified as a potential high-yield target for pharmaceutical prevention in treating many types of cancers (6).

Hepatocellular carcinoma (HCC) is the fifth most common cancer and the third leading cause of cancer-related mortality worldwide (7). The diagnosis of HCC is difficult due to the lack of early screening methods, and treatment is arduous due to its aggressive nature and the absence of therapeutic targets. Numerous studies regarding preventative and curative strategies for HCC have been conducted in recent years, leading to significant discoveries $(6,8,9)$. HCC patients are found to have high levels of IL- 6 that promote the survival of HCC cells through the upregulation of the STAT3 signaling pathway (10). Thus, abnormal levels of IL- 6 have profound impacts on cancer occurrence, development and progression. Heightened expression of IL- 6 may be blocked by disruption of the STAT3 pathway that in turn blocks cell transformation, inhibits angiogenesis and suppresses tumor growth (11); epigallocatechin-3-gallate (EGCG) can promote this disruption.

According to epidemiologic studies, the risk of $\mathrm{HCC}$, along with that of many types of cancers, can be reduced through tea consumption. Although green tea is a promising dietary source of chemopreventive and chemoprotective chemicals (12-14), its mechanism is still not fully understood. However, many 
reports have identified EGCG as the ingredient of green tea that contributes to the tea's anticancer function.

The purpose of this study was to gain insight into the molecular mechanism involved in the effects of STAT3. Surface plasmon resonance (SPR) detection, in silico docking simulations, MTT assay, FACS-based apoptosis assay, immunoblotting, and RT-PCR were among the techniques used to validate our findings. EGCG was found to disrupt Stat3-phosphorylated peptide binding, inhibit the expression of phosphorylated Stat3 protein as well as many downstream genes regulated by STAT3, induce HCC cell apoptosis, and suppress HCC cell growth, by possibly inhibiting the STAT3 signaling pathway to directly interfere with the Stat 3 protein.

\section{Materials and methods}

Reagents. EGCG was obtained from Catch Bio-Science \& Technology Ltd. (Jiangsu, China) with a purity of $>99.99 \%$. All cell culture reagents were purchased from Biowest (USA). MTT [3-(4,5-dimethylthiazol-2-yl)-2,5-diphenyl tetrazolium bromide] detection kit, Annexin V-FITC apoptosis detection kit and cell cycle detection kit were obtained from BestBio (Shanghai, China). Western blotting antibodies specific to p-Stat3, total-Stat3 and $\beta$-actin were purchased from Cell Signaling Technology, Inc. (Beverly, MA, USA). Cell-based ELISA kit [human/mouse phospho-Stat3 (Y705) immunoassay] was purchased from R\&D Systems (Minneapolis, MN USA).

Cell lines. The BEL-7402 and QGY-7703 human HCC cell lines were provided by Shanghai Institute of Biochemistry and Cell Biology. They were cultured in RPMI-1640 medium supplemented with $10 \%(\mathrm{v} / \mathrm{v})$ fetal bovine serum, $1 \mathrm{X}$ penicillin-streptomycin solution in a humidified $5 \% \mathrm{CO}_{2}$ atmosphere at $37^{\circ} \mathrm{C}$.

Stat3/pY-peptide binding assay. Stat 3 binding assays were performed at $25^{\circ} \mathrm{C}$ with a BIAcore 3000 biosensor using $20 \mathrm{mM}$ Tris-buffer $\mathrm{pH} 8.0$ that contained $2 \mathrm{mM} \beta$-mercaptoethanol and 5\% DMSO as running buffer. Phosphorylated and non-phosphorylated control biotinylated EGFR-derived dodecapeptides based on the sequence surrounding Y1068 were immobilized on a streptavidin-coated sensor chip (BIAcore Inc., Piscataway, NJ, USA). The binding of Stat3 was conducted in $20 \mathrm{mM}$ Tris-buffer $\mathrm{pH} 8.0$ containing $2 \mathrm{mM}$ $\beta$-mercaptoethanol at a flow rate of $10 \mu \mathrm{l} / \mathrm{min}$ for $1-2 \mathrm{~min}$. Aliquots of Stat 3 at $500 \mathrm{nM}$ were premixed with compound to achieve a final concentration of $1-1,000 \mu \mathrm{M}$, and incubated at $4^{\circ} \mathrm{C}$ prior to being injected onto the sensor chip. The chip was regenerated by injecting $10 \mu \mathrm{l}$ of $100 \mathrm{mM}$ glycine at $\mathrm{pH} 1.5$ after each sample injection. A control (Stat3 with DMSO but without compound) was run at the beginning and the end of each cycle (40 sample injections in total) to maintain the integrity of the sensor chip throughout the cycle run. The average of the 2 controls was normalized to $100 \%$ and used to evaluate the effect of each compound on Stat 3 binding. Responses were normalized by dividing the value at $2 \mathrm{~min}$ by the response obtained in the absence of compounds at $2 \mathrm{~min}$ and multiplying by $100 . \mathrm{IC}_{50}$ values were determined by plotting the percentage of the maximum response as a function of the log concentra- tion of the compound, and fitting the experimental points to a competitive binding model using a four-parameter logistic equation: $\mathrm{R}=\mathrm{R}_{\text {high }}-\left(\mathrm{R}_{\text {high }}-\mathrm{R}_{\text {low }}\right) /\left(1+\right.$ conc $\left./ \mathrm{A}_{1}\right) \mathrm{A}_{2}$, where $\mathrm{R}$ is the percent response at the inhibitor concentration; $R_{\text {high }}$ is the percent response with no compound; $\mathrm{R}_{\text {low }}$ is the percent response at the highest compound concentration; $\mathrm{A}_{2}$ is the fitting parameter (slope); and $\mathrm{A}_{1}$ is the $\mathrm{IC}_{50}$ (BIAevaluation software version 4.1).

Molecular docking between EGCG and Stat3. Both molecular structures of Stat 3 and EGCG were retrieved from the Protein Data Bank (PDB Code: 1BG1 and ENG5) and optimized in the implicit solvent for docking preparation. The docking region mainly constructed by the residues of Arg-609 and K-591 was localized based on the interface between the STAT3 SH2 domain and the phosphopeptide. The docking procedure was executed using CHARMm force field on the CDOCKER module platform in Discovery Studio (Accelrys Inc.). The conformation search space was limited to a spherical region with a center of $104.8,74.3,63.3$ and a radius of $13 \AA$. The other parameters were determined based on the default setting of the module, with a grid extension of 8.0. The ligand partial charge method was performed by CHARMm. Ten top hits were obtained from the docking simulation. Simulated annealing method was employed for the final conformation treatment (the system was heated to $700 \mathrm{~K}$ with 2,000 steps and then cooled to $300 \mathrm{~K}$ with 5,000 steps). Finally, the best conformation was selected as the analysis object according to the values of the scores.

Cell proliferation assay. Cell proliferation was determined using MTT assay according to the manufacturer's instructions. Briefly, BEL-7402 and QGY-7703 cells were then seeded into 96-well plates at a density of 5x103/well $(100 \mu \mathrm{l})$. After $24 \mathrm{~h}$, the indicated concentrations of EGCG were added. After incubation for 24 and $48 \mathrm{~h}$, respectively, cells were washed twice with PBS. Ten microliters of MTT medium was then added into each well, at which time cells were incubated for another $3 \mathrm{~h}$. The medium was removed, and $150 \mu \mathrm{l}$ of dissolution was added into each well. The plate was gently rotated on an orbital shaker for $10 \mathrm{~min}$ to dissolve the precipitate completely. The absorbance was detected at $492 \mathrm{~nm}$ with a microplate reader.

Flow cytometry and detection of apoptosis. QGY-7703 cells were treated with EGCG in complete medium for $48 \mathrm{~h}$ as previously described. Following treatment, the cells were harvested by trypsin (not containing EDTA) and rinsed twice with PBS at $4^{\circ} \mathrm{C}$. Cells were then resuspended in $1 \mathrm{X}$ Annexin $\mathrm{V}$ binding buffer. Five microliters of Annexin V-FITC solution was added to each tube. All tubes were incubated for $15 \mathrm{~min}$ at $4^{\circ} \mathrm{C}$ in darkness. Fifteen microliters of PI solution was added to each tube. All tubes were incubated for another $5 \mathrm{~min}$ at $4^{\circ} \mathrm{C}$ in darkness. Cells were then analyzed using flow cytometry (Accuri C6; BD Biosciences, USA).

Western blotting and ELISA. To detect protein expression and modification in response to treatment with EGCG, HCC cells, which were treated with various concentrations of EGCG, were plated onto 6 -well plates at a density of $2 \times 10^{5}$ cells $/ \mathrm{ml}$. After incubation for $24 \mathrm{~h}$, cells were lysed in cold RIPA lysis 

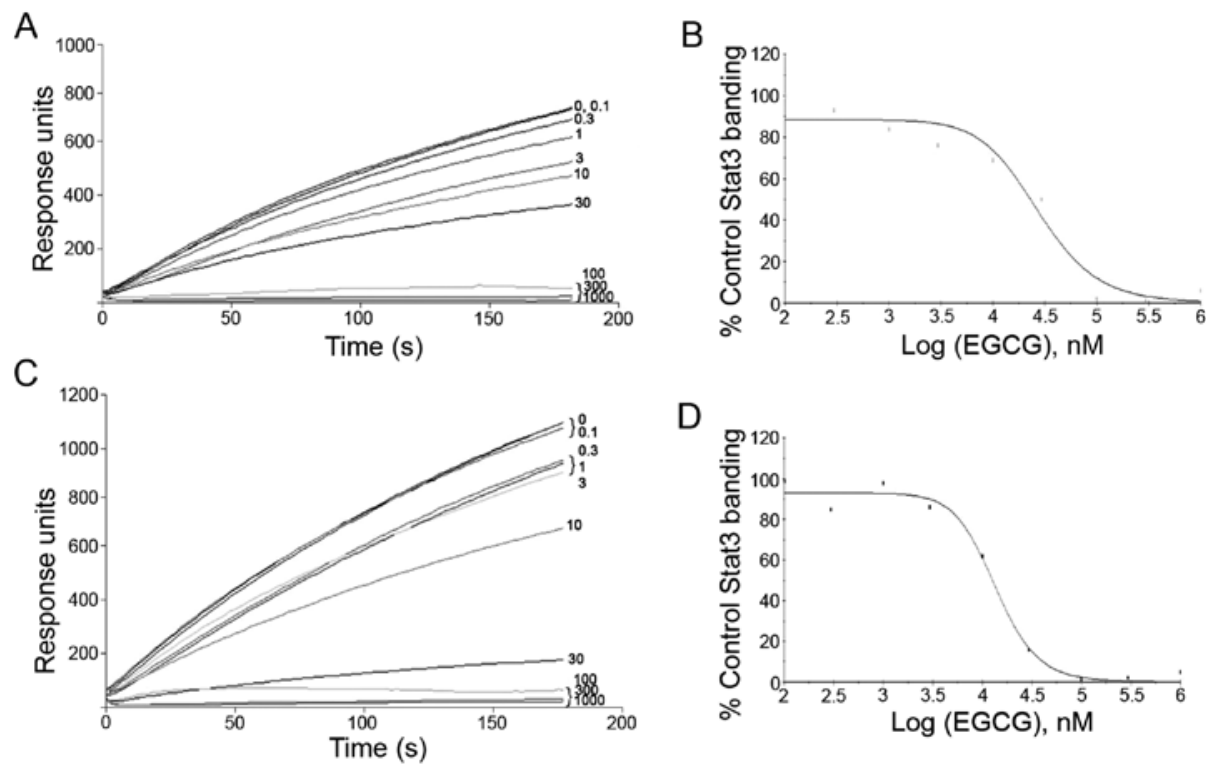

Figure 1. Inhibition of Stat3 binding to immobilized phosphopeptide ligand by EGCG. (A and C) Binding of recombinant Stat3 (500 nM) to a BIAcore sensor chip coated with a phosphopeptide that is based on amino acid sequence surrounding Y1068 within the EGFR was measured in real-time by SPR in the absence or presence of increasing concentrations $(0.1-1,000 \mu \mathrm{M})$ of EGCG. Data are response units as a function of time in seconds and are representative of 2 or more experiments. (B and D) The equilibrium binding levels obtained from the absence or presence of EGCG were normalized and plotted against the log concentrations of EGCG. The experimental points for EGCG were fit to competitive binding curves that used a four-parameter logistic equation. These curves were used to calculate the $\mathrm{IC}_{50}$ value for EGCG.

buffer. Total protein was extracted with high-salt buffer $(0.5 \%$ sodium deoxycholate, $1 \% \mathrm{SDS}, 1 \mathrm{mM}$ sodium orthovanadate, $1 \mathrm{mM} \beta$-glycerol phosphate, $1 \mathrm{mM}$ sodium fluoride, $2.5 \mathrm{mM}$ sodium pyrophosphate) containing a protease inhibitor cocktail (Roche, Nutley, NJ, USA). Protein samples were separated by SDS-PAGE, transferred onto PVDF membranes, and immunoblotted with the corresponding antibodies. The signals were visualized with Enhanced Chemiluminescence Plus (ECL Plus) detection system (Dingguo, China). The ELISA procedure was used according to the manufacturer's instructions (Cell Signaling Technology, Inc.). Briefly, $100 \mu \mathrm{l}$ of 15,000 BEL-7402 cells was seeded into each well of a black 96-well microplate with a clear bottom, and incubated overnight at $37^{\circ} \mathrm{C}$. Cells were then treated with different concentrations of EGCG in complete medium for $24 \mathrm{~h}$. Subsequently, cells were stimulated with interleukin 6 (IL-6) $(50 \mathrm{ng} / \mathrm{ml})$ to induce Stat 3 phosphorylation. Following the treatments, cells were treated and tested with the cell-based ELISA kit.

RT-PCR. The BEL-7402 and QGY-7703 cells were treated with EGCG at 40,80 and $160 \mu \mathrm{M}$ for $48 \mathrm{~h}$ as previously described. Total RNAs were extracted from the cells using a commercially available RNA-Bee isolation kit (Tel-Test). Standard reverse transcription was performed with $500 \mathrm{ng}$ of total RNA using TIANScriptRT kit (Tiangen Beijing, China). Reverse transcription-PCR was performed using $1 \mu \mathrm{l}$ of cDNA template, 10 pmol of primers, and a PCR premix (1 U Taq DNA polymerase, $250 \mathrm{mM}$ dNTPs, $10 \mathrm{mM}$ Tris- $\mathrm{HCl}, 40 \mathrm{mM}$ $\mathrm{KCl}$ and $1.5 \mathrm{mM} \mathrm{MgCl}_{2}$; Tiangen). The following primers were used in the PCR reactions: Bcl-xL forward, 5'-agctggtggt tgactttctctc-3' and reverse, 5'-ccggaagagttcattcactacc-3'; c-Myc forward, 5'-ctaccctctcaacgacagcag-3' and reverse, 5'-gtgtgtt cgcctcttgacatt-3'; VEGF forward, 5'-gcagaatcatcacgaagtggt-3' and reverse, 5'-catttgttgtgctgtaggaagc-3'; cyclin D1 forward, 5'-atctacaccgacaactccatcc-3' and reverse, 5'-gcattttggaga ggaagtgttc-3'; $\beta$-actin forward, 5'-agagctacgagctgcetgctg-3' and reverse, 5'-agtacttgcgctcaggagga-3'.

The amplified products obtained from the $\beta$-actin-specific primers served as internal controls. PCR was conducted using Bio-Rad T-100 (Bio-Rad, Hercules, CA, USA) with a 5-min denaturation step at $94^{\circ} \mathrm{C} ; 30$ cycles of $94^{\circ} \mathrm{C}$ for $30 \mathrm{sec}, 62^{\circ} \mathrm{C}$ for $30 \mathrm{sec}$ and $72^{\circ} \mathrm{C}$ for $30 \mathrm{sec}$; and a final extension at $72^{\circ} \mathrm{C}$ for $10 \mathrm{~min}$. PCR amplifications were verified to be in the linear range.

Statistical analysis. Data are presented as means \pm SD for 3 separate experiments. One-way ANOVA was employed for statistical analysis using SPSS 17.0. $\mathrm{P}<0.05$ was considered to indicate a statistically significant result.

\section{Results}

EGCG blocks Stat3 binding to its phosphopeptide ligand. EGCG was tested for its ability to block Stat3 binding to its phosphopeptide ligand using SPR binding assay (15). SPR experiments showed that EGCG was able to directly compete with pY-peptide for binding with Stat 3 at an $\mathrm{IC}_{50}$ value of 10-30 $\mu \mathrm{M}$ (Fig. 1).

Molecular docking between EGCG and Stat3. Fig. 2A is a computer model image of EGCG on Stat3. According to the figure, EGCG is located in a phosphopeptide binding pocket formed by the STAT3 $\mathrm{SH} 2$ fold. Fig. 2B shows the spatial matching results of EGCG and Stat3; the 3-D structure of EGCG matches perfectly with the phosphopeptide binding site of STAT3 SH2. Fig. 2C and D depict the specific 

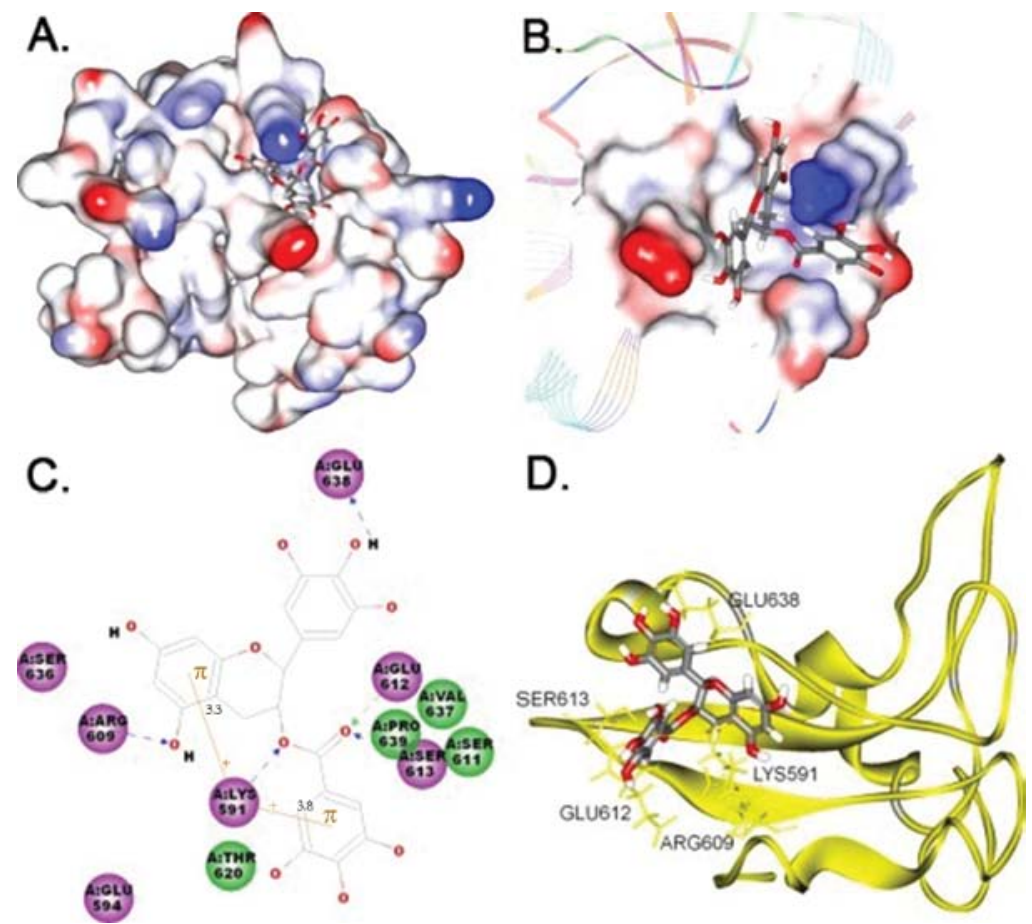

Figure 2. Computer modeling of EGCG bound by the SH2 domain of STAT3. The 3-D structures and results of computer docking of EGCG to the STAT3 SH2 domain are shown in A, B and D. The left side of C shows the 2-D structure. The middle portion of A and B shows EGCG binding to an electrostatic molecular surface model of the STAT3 SH2 domain; blue represents areas with positive-charge; red represents areas with negative-charge and the stick model depicts EGCG. $C$ is a closer view of this interaction with hydrogen bonds indicated by dotted lines. Cation- $\pi$ interactions are shown in brown color and critical residues that contribute mainly to the EGCG-Stat3 binding are represented by circles in green or purple colors, and EGCG molecules are presented by a line model. In D, STAT3 SH2 skeletal configuration is presented in a ribbon model, EGCG is represented by stick models and all atoms of critical residues in STAT3 SH2 domains are represented in gold color. The carbon, oxygen and hydrogen atoms of EGCG are represented by silver, red and white color, respectively, in A, B, $\mathrm{C}$ and $\mathrm{D}$.

\section{A}

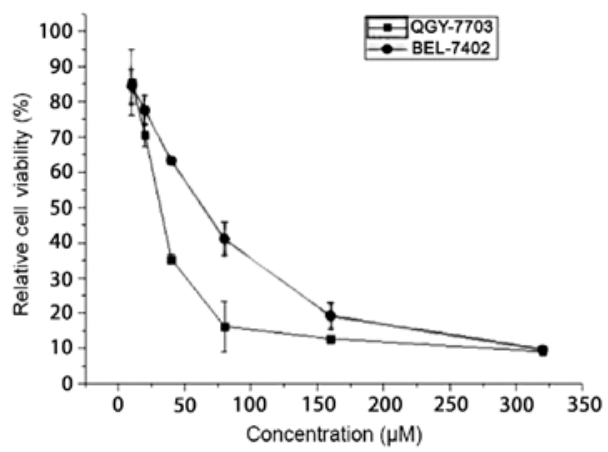

B

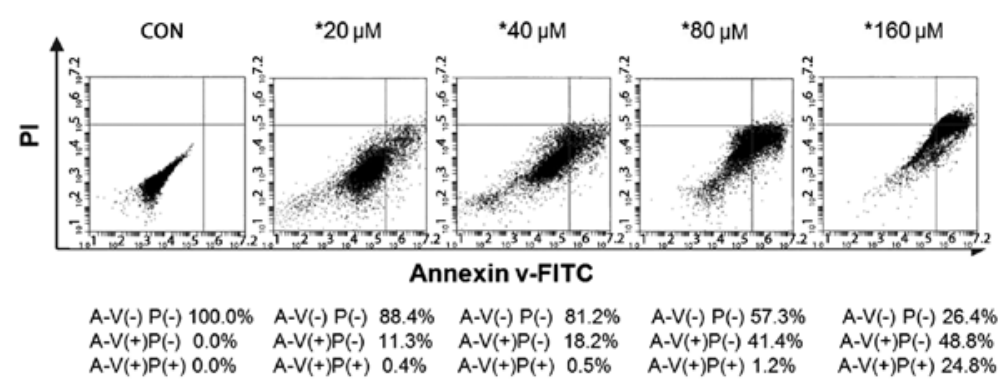

Figure 3. EGCG suppresses HCC cell proliferation and induces cell apoptosis. (A) QGY-7703 and BEL-7402 cell lines were treated with different concentrations of EGCG, and the relative cell viability was measured using MTT assay. The standard deviations were calculated based on 3 experiments. (B) QGY-7703 cells were cultured with different concentrations of EGCG for $48 \mathrm{~h}$, and the apoptotic fraction of cells was detected by Annexin V staining (x-axis)/PI (y-axis) staining using flow cytometry. The lower right quadrant of the plot indicates early apoptotic cells that are Annexin V-positive and PI-negative.

interactions between EGCG and STAT3 SH2. According to these two figures, the -NH3 group of LYS591 is located between the 2 aromatic rings of EGCG and the 2 hydrogen atoms from the $-\mathrm{NH} 3$ group, resulting in the formation of cation- $\pi$ bonds. The other hydrogen atom in the - $\mathrm{NH} 3$ group forms a hydrogen bond with the -O- in EGCG. On the other hand, the $-\mathrm{C}=\mathrm{O}$ group functions as a hydrogen receptor in EGCG, forming hydrogen bonds with -NH in GLU612 and -OH in SER613, respectively. While the 2 aromatic rings both function as hydrogen donors in EGCG, one also forms hydrogen bonds with the - $\mathrm{NH} 2$ group in ARG60, while the other forms hydrogen bonds with the -NH2 group in Glu638. The other part of EGCG forms a van der Waals interaction with the phosphopeptide binding pocket of STAT3 SH2 (LYS591, GLU594, ARG609, SER611, GLU612, SER613, THR620, VAL637, GLU638, PRO639) (Fig. 2C). These multiple interactions result in a steady locked relationship (15-18).

EGCG suppresses BEL-7402 and QGY-7703 cell growth, and induces apoptosis in QGY-7703 cells. To determine the potential cytotoxic and anti-proliferative effects of EGCG, the human HCC cell lines BEL-7402 and QGY-7703 were cultured with 
A

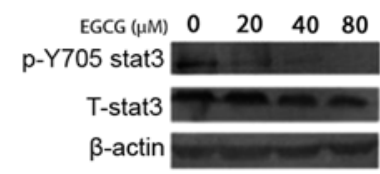

B

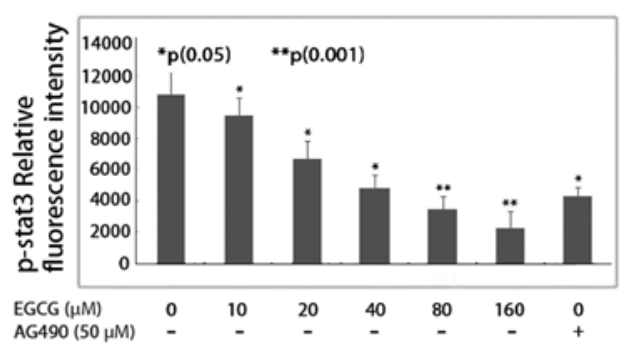

C Bel-7402 cell

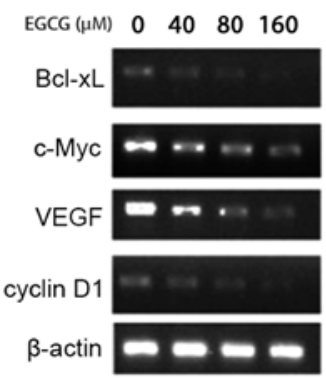

QGY-7703 cell

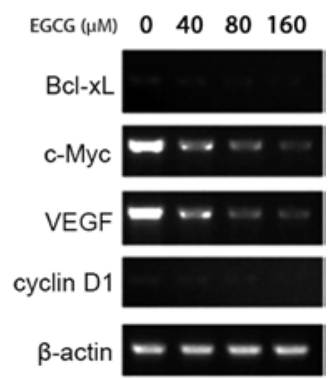

Figure 4. EGCG inhibits Stat3 phosphorylation and suppresses the expression of STAT3-regulated genes. (A) QGY-7703 cells were treated with EGCG $(0-80 \mu \mathrm{M})$ for $48 \mathrm{~h}$. The expression levels of $\mathrm{p}$-Stat 3 and T-Stat 3 were detected by western blotting. $\beta$-actin was used as a loading control. (B) The relative intensities of p-Stat 3 in QGY-7703 cells were measured by cell-based ELISA. Cells treated with AG490 were used as positive control. Data represent the means $\pm \mathrm{SD}$. ${ }^{*} \mathrm{P}<0.05$ or ${ }^{* *} \mathrm{P}<0.001$ indicates a significant difference from the respective control. (C) mRNA levels of Bcl-xL, c-Myc, VEGF and cyclin D1 in QGY-7703 cells treated with EGCG were detected by RT-PCR. Housekeeping gene $\beta$-actin was used as the control.

EGCG at various concentrations $(0-320 \mu \mathrm{M})$. Cell viability was then determined by MTT assay. Results showed that treatment with EGCG led to a significant dose-dependent inhibition of HCC cell growth in vitro (Fig. 3A). The half maximal $(50 \%)$ inhibitory concentrations $\left(\mathrm{IC}_{50}\right)$ for BEL-7402 and QGY-7703 cells were $\sim 55$ and $35 \mu \mathrm{M}$, respectively. Induction of cell apoptosis was confirmed by Annexin V-FITC staining in QGY-7703 cells. Results showed that treatment with EGCG led to significant dose-dependent apoptosis-inducing effects on HCC cell growth in vitro. According to Fig. 3B, the upper right quadrant represents late apoptosis, while the lower right quadrant represents early apoptosis. Increasing concentrations of EGCG at 20, 40, 80 and $160 \mu \mathrm{M}$, respectively, were added to the QGY-7703 cell line for $48 \mathrm{~h}$. As a result, the rates of cell apoptosis were 11.7, 18.7, 42.6 and $73.6 \%$, respectively. Thus, as the concentration of EGCG increased, the rate of apoptosis of the QGY-7703 cells also increased. The standard deviations were calculated based on 3 independent experiments.

EGCG inhibits IL-6-induced Stat-3 phosphorylation. To examine whether EGCG has inhibitory effects on IL-6induced Stat 3 phosphorylation, QGY-7703 cells were cultured

and pretreated with different concentrations of EGCG for $48 \mathrm{~h}$, and were then treated with $50 \mathrm{ng} / \mathrm{ml}$ of IL- 6 stimulation for $30 \mathrm{~min}$. After treatment, the phosphorylated Stat 3 and total Stat 3 were analyzed by western blotting and cell-based ELISA. EGCG inhibited Stat 3 phosphorylation on tyrosine 705 in a dose-dependent manner (Fig. 4A). The p-Stat 3 relative florescence intensity was significantly reduced following EGCG treatment. When QGY-7703 cells were treated with EGCG at concentrations of $10,20,40,80$ and $160 \mu \mathrm{M}$, respectively, the p-Stat 3 relative average fluorescence intensities were 7,400, $6,600,4,500,3,400$ and 2,200, respectively. Statistical analysis showed a P-value of $<0.05$ for EGCG at 10,20 and $40 \mu \mathrm{M}$ in relation to their corresponding fluorescence intensity; EGCG at 80 and $160 \mu \mathrm{M}$ had a $\mathrm{P}$-value of $<0.001$ in relation to their corresponding fluorescence intensity (Fig. 4B).

EGCG downregulates the expression of cancer-related genes. Exposure to EGCG resulted in a dose-dependent decrease in cyclin D1 mRNA expression in both BEL-7402 and QGY-7703 cells, as demonstrated by RT-PCR analysis. Furthermore, the expression levels of Bcl-xL, c-Myc and VEGF were also significantly reduced at the transcriptional levels (Fig. 4C).

\section{Discussion}

Tea is one of the most popular beverages in the world and has been well known to promote good health in numerous ways for over two thousand years. Daily consumption of tea may reduce cholesterol and the incidence of heart disease, boost immunity and benefit human skin. Particularly, tea may lower the risk of various types of cancers, including gastric, pancreatic and colorectal, in the human population (19-21). EGCG, which contributes to more than $40 \%$ of the total polyphenol mixture in tea, plays an essential role in its chemotherapeutic and chemopreventive effects. In fact, the anti-oxidative activity and metal chelating functions of EGCG may contribute to the inhibitory activity of tea against carcinogenesis (22). Additionally, there is considerable evidence that EGCG has an anticancer nature by modulating the intracellular signaling network.

To study the mechanism of the inhibitory effects of EGCG on carcinoma cells, we conducted molecular binding computation and related experiments. Based on our study from the BIAcore binding assay in micromoles, EGCG blocked Stat3 binding to its phosphopeptide ligand on SPR testing. Furthermore, the EGCG molecule had major interactions with two key residues, R609 and K591, localized in the STAT3 SH2 domain, which we found through docking simulation analysis. We then confirmed that EGCG significantly inhibited carcinoma cell growth in vitro in two human HCC cell lines, BEL-7402 and QGY-7703, in a dose-dependent trend by MTT assay. Additionally, EGCG interrupted Stat3 phosphorylation on tyrosine 705 in a dose-dependent manner as detected by western blotting and cell-based ELISA immunoblot testing in micromoles. As shown in Fig. 4B, EGCG at $40 \mu \mathrm{M}$ had the same effects on $\mathrm{p}$-Stat 3 phosphorylation inhibition as the wellknown EGFR inhibitor AG490 at $50 \mu \mathrm{M}$. HCC cells treated with EGCG exhibited a significant transcriptional decrease in the expression of many genes related to cell growth, survival and apoptosis, including Bcl-xL, c-Myc, VEGF and cyclin D1, 
as determined by RT-PCR analysis. This in turn led to HCC cell apoptosis, as demonstrated by flow cytometry.

Our research data support that the anticancer function of green tea is the result of the inhibition of the STAT3 signaling pathway by EGCG. However, additional studies suggest that EGCG is not only a multiple effector that regulates cell signaling such as STAT1 and ERK1/2 (23), but is also a general binder that binds to STAT1 and other bio-molecules, including RNA. Based on our conclusion, EGCG is a STAT3 signaling inhibitor that competitively binds to the STAT3 SH2 domain, contributing to the regulation of the cellular signaling network and the anticancer effects of green tea. However, further research is needed before a full understanding of the mechanism of EGCG in tea is achieved in order to benefit the health of the general population.

\section{Acknowledgements}

We thank Shanghai Institute of Biochemistry and Cell Biology for contributing the BEL-7402 and QGY-7703 human HCC cell lines.

\section{References}

1. Darnell JE Jr, Kerr IM and Stark GR: Jak-STAT pathways and transcriptional activation in response to IFNs and other extracellular signaling proteins. Science 264: 1415-1421, 1994.

2. Buettner R, Mora LB and Jove R: Activated STAT signaling in human tumors provides novel molecular targets for therapeutic intervention. Clin Cancer Res 8: 945-954, 2002.

3. Aggarwal BB, Sethi G, Ahn KS, Sandur SK, Pandey MK, Kunnumakkara AB, Sung B and Ichikawa H: Targeting signaltransducer-and-activator-of-transcription-3 for prevention and therapy of cancer: modern target but ancient solution. Ann NY Acad Sci 1091: 151-169, 2006.

4. Redell MS and Tweardy DJ: Targeting transcription factors in cancer: challenges and evolving strategies. Drug Discov Today Technol 3: 261-267, 2006.

5. Whittaker S, Marais R and Zhu AX: The role of signaling pathways in the development and treatment of hepatocellular carcinoma. Oncogene 29: 4989-5005, 2010.

6. Berishaj M, Gao SP, Ahmed S, Leslie K, Al-Ahmadie H, Gerald WL, Bornmann W and Bromberg JF: Stat 3 is tyrosinephosphorylated through the interleukin-6/glycoprotein 130/ Janus kinase pathway in breast cancer. Breast Cancer Res 9: R32, 2007.

7. Lin L, Amin R, Gallicano GI, Glasgow E, Jogunoori W, Jessup JM, Zasloff M, Marshall JL, Shetty K, Johnson L, Mishra L and He AR: The STAT3 inhibitor NSC 74859 is effective in hepatocellular cancers with disrupted TGF- $\beta$ signaling. Oncogene 28: 961-972, 2009.

8. Soresi M, Giannitrapani L, D'Antona F, Florena AM, La Spada E, Terranova A, Cervello M, D'Alessandro N and Montalto G: Interleukin-6 and its soluble receptor in patients with liver cirrhosis and hepatocellular carcinoma. World J Gastroenterol 12: 2563-2568, 2006.
9. Berasin C, Castillo J, Perugorria MJ, Latasa MU, Prieto J and Avila MA: Inflammation and liver cancer: new molecular links. Ann NY Acad Sci 1155: 206-221, 2009.

10. Calvisi DF, Ladu S, Gorden A, Farina M, Conner EA, Lee JS Factor VM and Thorgeirsson SS: Ubiquitous activation of Ras and Jak/Stat pathways in human HCC. Gastroenterology 130: $1117-1128,2006$.

11. To KF, Chan MW, Leung WK, Ng EK, Yu J, Bai AH, Lo AW, Chu SH, Tong JH, Lo KW, Sung JJ and Chan FK: Constitutional activation of IL-6-mediated JAK/STAT pathway through hypermethylation of SOCS-1 in human gastric cancer cell line. Br J Cancer 91: 1335-1341, 2004.

12. Li HC, Yashiki S, Sonoda J, Lou H, Ghosh SK, Byrnes JJ, Lema C, Fujiyoshi T, Karasuyama M and Sonoda S: Green tea polyphenoles induce apoptosis in vitro in peripheral blood T lymphocytes of adult T-cell leukemia patients. Jpn J Cancer Res 91: 34-40, 2000.

13. Park G, Yoon BS, Moon JH, Kim B, Jun EK, Oh S, Kim H, Song HJ, Noh JY, Oh C and You S: Green tea polyphenol epigallocatechin3-gallate suppresses collagen production and proliferation in keloid fibroblasts via inhibition of the STAT3-signaling pathway. J Invest Dermatol 128: 2429-2441, 2008.

14. Ahn HY, Hadizadeh KR, Seul C, Yun YP, Vetter H and Sachinidis A: Epigallocathechin-3 gallate selectively inhibits the PDGF-BB-induced intracellular signaling transduction pathway in vascular smooth muscle cells and inhibits transformation of sis-transfected NIH 3T3 fibroblasts and human glioblastoma cells. Mol Biol Cell 10: 1093-1104, 1999.

15. Xu X, Kasembeli MM, Jiang X, Tweardy BJ and Tweardy DJ: Chemical probes that competitively and selectively inhibit Stat3 activation. PLoS One 4: e4783, 2009.

16. Kasembeli MM, Xu X and Tweardy DJ: SH2 domain binding to phosphopeptide ligands: potential for drug targeting. Front Biosci 14: 1010-1022, 2009

17. Shao H, Xu X, Jing N and Tweardy DJ: Unique structural determinants for Stat3 recruitment and activation by the granulocyte colony-stimulating factor receptor at phosphotyrosine ligands 704 and 744. J Immunol 176: 2933-2941, 2006.

18. Shao H, Xu X, Mastrangelo MA, Jing N, Cook RG, Legge GB and Tweardy DJ: Structural requirements for signal transducer and activator of transcription 3 binding to phosphotyrosine ligands containing the YXXQ motif. J Biol Chem 279: 18967-18973, 2004.

19. Takada M, Nakamura Y, Koizumi T, Toyama H, Kamigaki T, Suzuki Y, Takeyama Y and Kuroda Y: Suppression of human pancreatic carcinoma cell growth and invasion by epigallocatechin-3-gallate. Pancreas 25: 45-48, 2002.

20. Zhu B, Chen H, Zhan W, Wang CY, Cai SR, Wang Z, Zhang CH and He YL: (-)-Epigallocatechin-3-gallate inhibits VEGF expression induced by IL- 6 via Stat 3 in gastric cancer. World $\mathbf{J}$ Gastroenterol 7: 2315-2325, 2011.

21. Yang GY, Liao J, Kim K, Yurkow EJ and Yang CS: Inhibition of growth and induction of apoptosis in human cancer cell lines by tea polyphenols. Carcinogenesis 19: 611-616, 1998.

22. Yang CS, Lambert JD, Hou Z, Ju J, Lu G and Hao X: Molecular targets for the cancer preventive activity of tea polyphenols. Mol Carcinog 45: 431-435, 2006.

23. Shankar S, Suthakar G and Srivastava RK: Epigallocatechin3-gallate inhibits cell cycle and induces apoptosis in pancreatic cancer. Front Biosci 12: 5039-5051, 2007. 